\title{
Inversão 1D de dados MMT de Ambientes Altamente Resistivos em Águas Profundas
}

\author{
Frayzer Lima de Almeida, UFPA, Brazil
}

Copyright 2014, SBGf - Sociedade Brasileira de Geofísica

Este texto foi preparado para a apresentação no VI Simpósio Brasileiro de Geofísica, Porto Alegre, 14 a 16 de outubro de 2014. Seu conteúdo foi revisado pelo Comitế Técnico do VI SimBGf, mas não necessariamente representa a opinião da SBGf ou de seus associados. É proibida a reprodução total ou parcial deste material para propósitos comerciais sem prévia autorização da SBGf

\section{Resumo}

Inicialmente apresentamos a formulação unidimensional do método magnetotelúrico marinho - MMT 1D, para em seguida propormos a modelagem inversa de dados do MMT 1D segundo a função objetivo normalizada juntamente com vínculos absolutos e minimizada através do método de Marquardt. Os modelos geoelétricos usados para simulação dos dados sintéticos (acrescidos com ruído) referem-se a ambientes altamente resistivos característicos das bacias costeiras brasileiras encontras na exploração de hidrocarbonetos. Os resultados de inversão através da metodologia acima proposta são comparados com os resultados segundo uma das metodologias bastante difundida na literatura de inversão geofísica, que consiste na minimização da função objetivo usual juntamente com vínculos absolutos através do método de Marquartd.

\section{Introdução}

A partir de 2000 os cenários financeiro e técnico, na exploração de hidrocarbonetos, mudaram. O financeiro é que nos últimos anos o preço do petróleo vem crescendo extraordinariamente, isto favorece a incursão de novas técnicas não-sísmica para auxiliar a sísmica na detecção de hidrocarbonetos e, com isso, minimizar o risco exploratório, que ainda é bastante alto. Já o cenário técnico é que a partir do ano 2000 o campo para a exploração de hidrocarbonetos se expandiu para regiões de águas profundas e ultra-profundas em ambientes altamente resistivos, onde o preço de um poço exploratório de petróleo é exorbitantemente alto (Rijo, 2007), exigindo tanto o auxílio de novas técnicas nãosísmica na exploração de hidrocarbonetos quanto técnicas avançadas de engenharia na perfuração e na extração de hidrocarbonetos nestes novos campos.

Devido a estas duas condições favoráveis, os métodos geofísicos elétricos e eletromagnéticos - EM apropriados e indicados na prospecção de hidrocarbonetos em águas profundas e ultra-profundas são 0 método Eletromagnético Marinho de Fonte Controlada - mCSEM e o método Magnetotelúrico Marinho - MMT. São métodos eletromagnéticos que vêm se destacando na prospecção de hidrocarbonetos em águas profundas e ultra-profundas na última década, pois usados em conjunto à sísmica podem confirmar a existência de hidrocarbonetos, mapear o contorno de um reservatório e contribuir para a diminuição da ambigüidade na interpretação geológica.
O método MMT vem sendo indicado e muito utilizado para auxiliar na investigação da existência de possíveis reservatórios de hidrocarbonetos em ambientes altamente resistivos localizados em águas profundas e ultra-profundas como, por exemplo, no Golfo do México e em bacias costeiras no Brasil, ou mais especificamente, os ambientes geológicos constituintes do pré-sal brasileiro. Neste caso o MMT é mais indicado que o mCSEM, uma vez que este último não é capaz de mapear a base da estrutura de sal devido suas medidas serem afetadas pela variação de resistividade interna desta estrutura, em conjunto com a resistividade de um possível reservatório de hidrocarbonetos existente em sua base. Esta característica de limitação se deve também ao fato do mCSEM e do imageamento sísmico em profundidade, em vários casos de interesse, não serem capazes de distinguir entre as estruturas de sal com raízes verticais profundas das que não são profundas.

Primeiramente é apresentada uma breve formulação do método MMT 1D. Secundariamente propomos uma metodologia para a inversão de dados 1D do MMT, a qual foi proposta por Almeida e Rijo (2011), porém para a inversão de dados 3D do mCSEM. Finalmente apresentamos quatro modelos geoelétricos de ambientes altamente resistivos em regiões de águas profundas e ultra-profundas, os quais são aplicados no processo de inversão segundo a metodologia acima proposta.

\section{O Problema direto}

A fonte para o MMT é a mesma para o método magnetotelúrico - MT, ou seja, são as ondas planas oriundas basicamente de interações entre os ventos solares, o campo magnético terrestre e a ionosfera. Os receptores para o MMT são afixados no assoalho marinho (contato mar e sedimentos) onde os mesmos medem, no campo, as componentes cartesianas $E_{x}$ e $H_{y}$, dos campos elétrico e magnético respectivamente, e por meio da seguinte fórmula:

$$
\rho_{a}=\frac{1}{\omega \mu_{0}}\left|\frac{E_{x}}{H_{y}}\right|^{2}
$$

defini-se a função $\rho_{a}$, denominada de resistividade aparente (em ohm-m). Esta resistividade aparente depende das resistividades elétricas do ambiente geoelétrico, das espessuras das camadas, de suas profundidades e da frequência, isto para a formulação unidimensional do MMT, (Rijo, 2001). A razão entre as componentes $E_{x}$ e $H_{y}$ resulta em um número complexo que em geofísica dos métodos elétricos e magnéticos - EM é denominado de impedância aparente 
na superfície onde se realizam os registros da sondagem do método MMT. Sendo assim, esta impedância possui um argumento o qual é denominado de fase da impedância na superfície de sondagem. Portanto, além da resistividade aparente $\rho_{a}$ usa-se também no método do MMT o valor, $\phi$, da fase da impedância na superfície de sondagem (assoalho marinho) que é dado em graus.

\section{O problema inverso}

Agora, o problema da inversão geofísica, aqui considerado e proposto, consiste na minimização da função objetivo normalizada:

$$
U\left(\mathbf{p}^{s}\right)=\sum_{k=1}^{2 n}\left(\omega_{k}-\phi_{k}\left(\mathbf{p}^{s}\right)\right)^{2}+\ell \sum_{j=1}^{q}\left(t_{i(j)}^{s}-\gamma_{i(j)}\right)^{2}
$$

onde $\omega_{k}=y_{k}^{o b s} / y_{k}^{o b s}=1 \mathrm{e} \phi_{k}\left(\mathbf{p}^{s}\right)=f_{k}\left(\mathbf{p}^{s}\right) / y_{k}^{o b s}$ desde que $y_{k}^{o b s}>0$ ou $y_{k}^{o b s}<0, k=1, \ldots, 2 n$.

$\mathrm{Na}$ Eq.(10) $\mathbf{p}^{s}=\left(p_{1}^{s}, p_{2}^{s}, \ldots, p_{m}^{s}\right)$ é o vetor dos parâmetros geoelétricos parametrizados (Almeida e Rijo, 2011), segundo $p_{i}^{s}=10^{\ln \left(\left|t_{i}^{s}\right|\right)}$ com $i=1,2,3, \ldots, m$ (desde que $p_{i}^{s}>0$ ), na s-ésima iteração. O vetor das variáveis paramétricas na s-ésima iteração é dado por $\mathbf{t}^{s}=\left(t_{1}^{s}, t_{2}^{s}, \ldots, t_{m}^{s}\right)$, sendo $m$ o número de parâmetros geoelétricos, $n$ o número de observações com $n>m$ e $y_{k}^{o b s}$ as componentes do vetor $\mathbf{y}^{o b s}$ dos dados observados, tanto da resistividade aparente quanto da fase, provenientes dos receptores localizados no assoalho marinho segundo o método MMT. As componentes $f_{k}\left(\mathbf{p}^{s}\right)$ são do vetor $\mathbf{f}\left(\mathbf{p}^{s}\right)$, na s-ésima iteração, com $k=1, \ldots, 2 n$, tanto da resistividade aparente quanto da fase proveniente da modelagem direta do MMT 1D, também na s-ésima iteração. Para cada período e/ou frequência, o método MMT fornece um valor da resistividade aparente e um valor da fase, logo para $n$ períodos do referido método tem-se $n$ resistividades aparentes e $n$ fases, daí de se ter $2 n$ no primeiro somatório da Eq.(2). O parâmetro $\ell$ é o multiplicador de Lagrange e $q$ é o número de parâmetros vinculados. $O$ vetor $\gamma=\left(\gamma_{i(j)}\right)$ com $i \in\{1,2,3, \ldots, m\}$ e $j=1,2, \ldots, q$ contém os vínculos absolutos paramétricos.

Já o problema de inversão geofísica, usual na literatura, consiste na minimização da seguinte função objetivo:

$$
U\left(\mathbf{p}^{s}\right)=\sum_{k=1}^{2 n}\left(y_{k}^{o b s}-f_{k}\left(\mathbf{p}^{s}\right)\right)^{2}+\ell \sum_{j=1}^{q}\left(p_{i(j)}^{s}-\beta_{i(j)}\right)^{2}
$$

onde $y_{k}^{o b s}$ são as componentes do vetor $\mathbf{y}^{o b s}$ dos dados observados, tanto da resistividade aparente quanto da fase, provenientes dos receptores localizados no assoalho marinho e as componentes $f_{k}\left(\mathbf{p}^{s}\right)$ são do vetor $\mathbf{f}\left(\mathbf{p}^{s}\right)$, na s-ésima iteração, com $k=1, \ldots, 2 n$, tanto da resistividade aparente quanto da fase provenientes da modelagem direta do MMT 1D acima descrita, também na s-ésima iteração.

O vetor $\quad \boldsymbol{\beta}=\left(\beta_{i(j)}\right) \quad$ com $\quad i \in\{1,2,3, \ldots, m\} \quad \mathrm{e}$ $j=1,2, \ldots, q$ contém os vínculos absolutos. Por exemplo, digamos que sabemos os valores dos parâmetros geoelétricos $p_{2}, p_{8}$ e $p_{m}$ os quais desejamos vinculá-los. Logo, $q=3$, e assim tem-se $\beta_{2(1)}:=p_{2}, \beta_{8(2)}:=p_{8}$ e $\beta_{m(3)}:=p_{m}$. Por tanto, o vetor dos vínculos absolutos é dado por $\boldsymbol{\beta}=\left(0, \beta_{2(1)}, 0,0,0,0,0, \beta_{8(2)}, 0, \ldots, 0, \beta_{m(3)}\right)$.

O processo de minimização tanto da Eq.(2) quanto da Eq.(3) é baseado no método de Marquardt (Press et al., 1992), juntamente com a estratégia proposta por Medeiros e Silva (1996) e Luiz (1999), que é exatamente a estratégia de introdução de informação a priori e que para o presente problema de inversão é especificamente chamada de introdução de vínculos absolutos. Para o maior entendimento tanto do método de Marquardt, como do algoritmo aqui aplicado na minimização das Eqs. (2) e (3) e do seu critério de parada, recomendamos Almeida e Rijo (2011).

\section{Modelos propostos}

Inicialmente vamos apresentar quatro modelos geoelétricos em ambientes altamente resistivos na prospecção de hidrocarbonetos em águas profundas e ultra-profundas do MMT 1D, segundo as características das bacias brasileiras offshore. Sendo que tais modelos serão submetidos à inversão geofísica EM, através da minimização tanto da função objetivo usual na literatura geofísica EM, Eq.(3), quanto da função objetivo normalizada proposta por Almeida e Rijo (2011), Eq.(2), ambas minimizadas pelo método de Marquardt (Press et al., 1992).

O primeiro modelo geoelétrico para o MMT 1D, aqui proposto (Fig.1), segundo características altamente resistivas em águas profundas é formado pela camada de ar com resistividade elétrica $\rho_{0}$ infinita. A camada do mar, com resistividade $\rho_{1}$ igual a 0,33 ohm-m e espessura $h_{1}$ igual a $2050 \mathrm{~m}$. A camada das rochas sedimentares encaixantes (folhelho, siltito, etc), com resistividade $\rho_{2}$ igual a 1,3 ohm-m e espessura $h_{2}$ igual a $1600 \mathrm{~m}$. A camada de sal (rochas sedimentares salinocarbonáticas) com resistividade $\rho_{3}$ igual a 45 ohm-m e espessura $h_{3}$ igual a $1500 \mathrm{~m}$. Já a camada plano paralela, a qual representa o reservatório de hidrocarbonetos, Fig.1, possui resistividade $\rho_{4}$ igual a 
25 ohm-m, e uma espessura $h_{4}$ igual a 750 metros. Finalmente a camada das rochas sedimentares encaixantes (semi-espaço inferior) apresenta resistividade $\rho_{5}$ igual a 3,0 ohm-m.

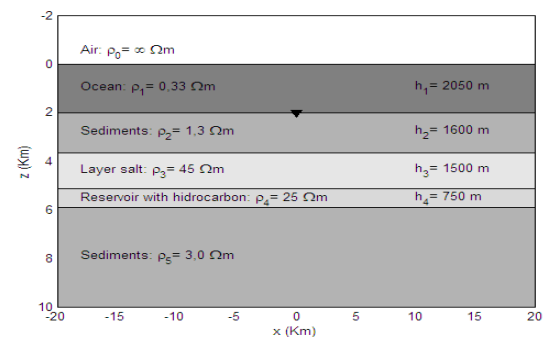

Figura $1-1^{\circ}$ modelo geoelétrico $1 D$ com camada salina possuindo espessura $h_{3}$ igual a 1500 metros, e camada reservatório de hidrocarbonetos possuindo uma espessura $h_{4}$ igual a $750 \mathrm{~m}$.

O segundo modelo geoelétricos para o MMT 1D, também proposto, segundo características altamente resistivas em águas profundas é formado pela camada de ar com resistividade elétrica $\rho_{0}$ infinita. A camada do mar, com resistividade $\rho_{1}$ igual a 0,33 ohm-m e espessura $h_{1}$ igual a $2000 \mathrm{~m}$. A camada das rochas sedimentares encaixantes (folhelho, siltito, etc), com resistividade $\rho_{2}$ igual a 1,0 ohm-m e espessura $h_{2}$ igual a $1000 \mathrm{~m}$. A camada de sal (rochas sedimentares salinocarbonáticas) com resistividade $\rho_{3}$ igual a $100 \mathrm{ohm}-\mathrm{m}$ e espessura $h_{3}$ igual a $2000 \mathrm{~m}$. Agora a camada plano paralela, a qual representa o reservatório de hidrocarbonetos para 0 modelo geoelétrico proposto apresenta resistividade $\rho_{4}$ igual a $50 \mathrm{ohm}$, e espessuras $h_{4}$ igual a 2000 metros. Finalmente a camada das rochas sedimentares encaixantes (semi-espaço inferior) apresenta resistividade $\rho_{5}$ igual a $1,0 \mathrm{ohm}-\mathrm{m}$.

O terceiro modelo geoelétrico para o MMT 1D, aqui proposto (Fig.2), segundo características altamente resistivas em águas ultra-profundas é formado pela camada de ar com resistividade elétrica $\rho_{0}$ infinita. A camada do mar, com resistividade $\rho_{1}$ igual a $0,33 \mathrm{ohm}$ m e espessura $h_{1}$ igual a $2000 \mathrm{~m}$. A camada das rochas sedimentares encaixantes (folhelho, siltito, etc), com resistividade $\rho_{2}$ igual a 1,0 ohm-m e espessura $h_{2}$ igual a $3000 \mathrm{~m}$. A camada de sal (rochas sedimentares salinocarbonáticas) com resistividade $\rho_{3}$ igual a 100 ohm-m e espessura $h_{3}$ igual a $2000 \mathrm{~m}$. Já a camada plano paralela, a qual representa o reservatório de hidrocarbonetos, Fig.2, possui resistividade $\rho_{4}$ igual a 50 ohm-m, e uma espessura $h_{4}$ igual a 1500 metros. Finalmente a camada das rochas sedimentares encaixantes (semi-espaço inferior) apresenta resistividade $\rho_{5}$ igual a $1,0 \mathrm{ohm}-\mathrm{m}$.

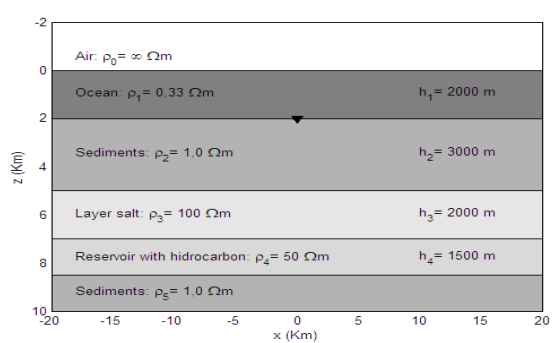

Figura $2-3^{\circ}$ modelo geoelétrico $1 D$ com uma camada salina à uma profundidade em relação ao assoalho marinho de 3000 metros e uma camada reservatório de hidrocarbonetos com uma espessura $h_{4}$ igual a 1500 metros.

Finalmente o quarto modelo geoelétrico para o MMT 1D, aqui proposto, é formado pela camada de ar com resistividade elétrica $\rho_{0}$ infinita. A camada do mar, com resistividade $\rho_{1}$ igual a 0,33 ohm-m e espessura $h_{1}$ igual a $2000 \mathrm{~m}$. A camada das rochas sedimentares encaixantes (folhelho, siltito, etc), com resistividade $\rho_{2}$ igual a 1,0 ohm-m e espessura $h_{2}$ igual a $1000 \mathrm{~m}$. A camada de sal (rochas sedimentares salinocarbonáticas) com resistividade $\rho_{3}$ igual a $100 \mathrm{ohm}-\mathrm{m}$ e espessura $h_{3}$ igual a $2000 \mathrm{~m}$. Já a camada plano paralela, a qual representa o reservatório de hidrocarbonetos possui resistividade $\rho_{4}$ igual a $90 \mathrm{ohm}-\mathrm{m}$, e uma espessura $h_{4}$ igual a 1500 metros. Finalmente a camada das rochas sedimentares encaixantes (semi-espaço inferior) apresenta resistividade $\rho_{5}$ igual a $1,0 \mathrm{ohm}-\mathrm{m}$.

\section{Resultados de inversão}

Realizamos inversão de dados do MMT 1D referente aos quatro modelos geoelétricos acima descritos. Onde cada conjunto de dados do MMT 1D (os dados observados das resistividades aparentes e das fases registrados nos receptores localizados no assoalho marinho) está relacionado a 21 períodos entre $10^{0}$ e $10^{4}$ segundos. Desta forma, para realizarmos a inversão dos dados do MMT 1D, implementamos ao código computacional, tanto a função objetivo usual na literatura geofísica EM (Eq.3) quanto a função objetivo normalizada (Eq.2) proposta por Almeida e Rijo (2011), ambas a serem minimizadas segundo o método de Marquardt (Press et al.,1992). Para um melhor detalhamento do algoritmo de inversão segundo o método de Marquardt na minimização da função objetivo normalizada, Eq.2, indica-se Almeida e Rijo (2011).

Comum a todos os processos de inversão tem-se os seguintes vínculos absolutos: a espessura da primeira camada (mar) e sua resistividade elétrica, a resistividade elétrica da segunda camada e a resistividade elétrica da quinta e última camada. Também comum a todos os processos de inversão, admitiu-se empiricamente o parâmetro de Marquardt igual a 0,1 e o multiplicador de Lagrange igual a 70. Agora, para serem realizadas as reconstituições e/ou inversões geofísica referentes aos 
modelos acima, foram realizadas as simulações destes modelos propostos a fim de se adquirir tanto as resistividades aparentes quanto as fases capturadas pelos receptores localizados no assoalho marinho. A estas resistividades aparentes foram acrescidos $7 \%$ de ruído gaussiano e nas fases foram acrescidos $3 \%$ de ruído gaussiano. Esta etapa consiste na criação dos dados observados sintéticos, a serem aplicadas nas reconstituições e/ou inversão geofísica aqui proposta.

\section{Inversão do Primeiro Modelo Geoelétrico}

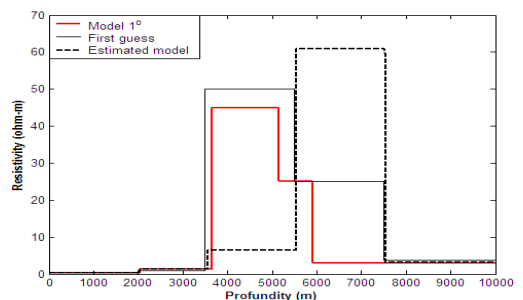

Figura 3: Resultado da inversão sobre o primeiro modelo geoelétrico (Fig.1), referente à minimização da função objetivo usual (Eq.3).

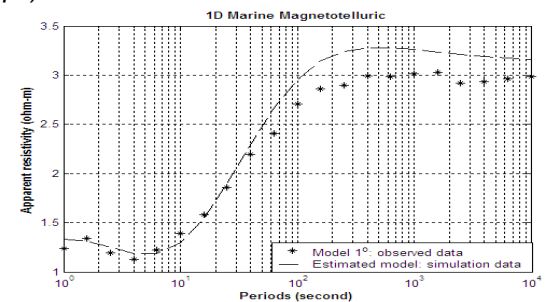

Figura 4: Resistividades aparentes do MMT $1 D$ simuladas tanto através do primeiro modelo geoelétrico (Fig.1), quanto pelo modelo estimado, referente à minimização da função objetivo usual (Eq.3).

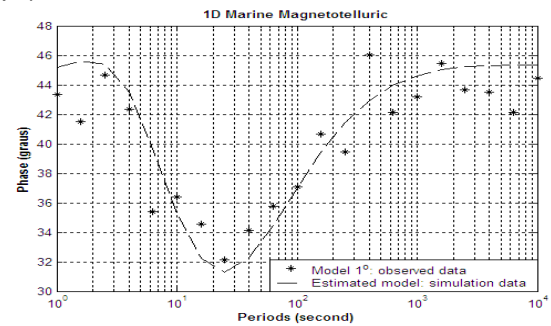

Figura 5: Fases do MMT 1D simulados tanto através do primeiro modelo geoelétrico (Fig.1), quanto pelo modelo estimado, referente à minimização da função objetivo usual (Eq.3).

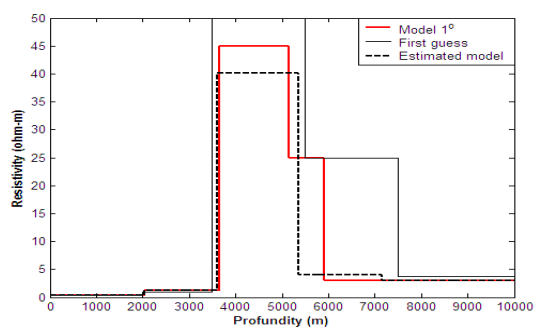

Figura 6: Resultado da inversão sobre o primeiro modelo geoelétrico (Fig.1), referente à minimização da função objetivo normalizada (Eq.2).

\section{Inversão do Segundo Modelo Geoelétrico}

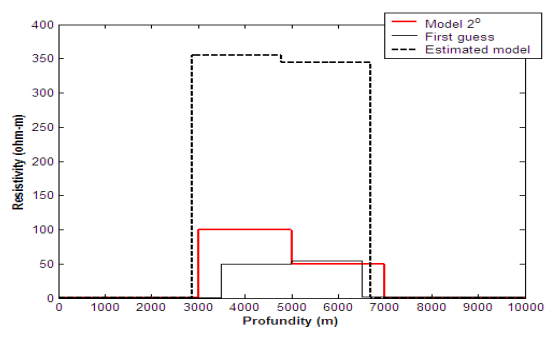

Figura 7: Resultado da inversão sobre o segundo modelo geoelétrico, referente à minimização da função objetivo usual (Eq.3).

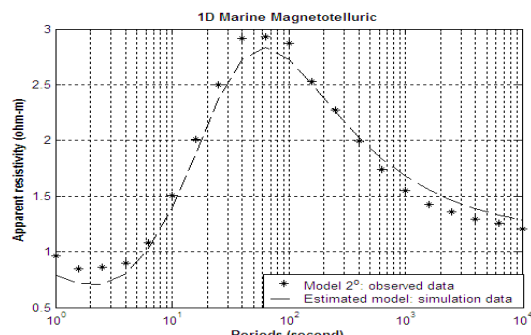

Figura 8: Resistividades aparentes do MMT 1D simuladas tanto através do segundo modelo geoelétrico, quanto pelo modelo estimado, referente à minimização da função objetivo usual (Eq.3).

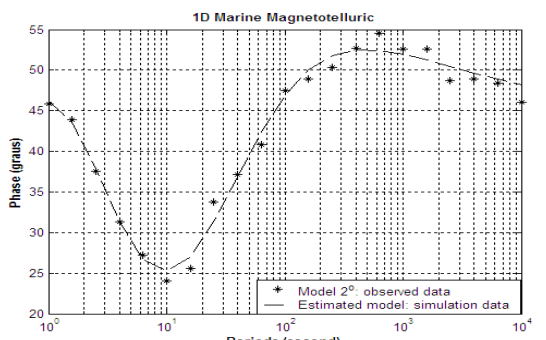

Figura 9: Fases do MMT $1 D$ simulados tanto através do segundo modelo geoelétrico, quanto pelo modelo estimado, referente à minimização da função objetivo usual (Eq.3).

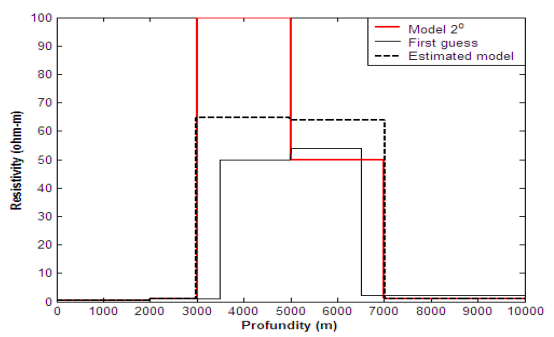

Figura 10: Resultado da inversão sobre o segundo modelo geoelétrico, referente à minimização da função objetivo normalizada (Eq.2). 


\section{Inversão do Terceiro Modelo Geoelétrico}

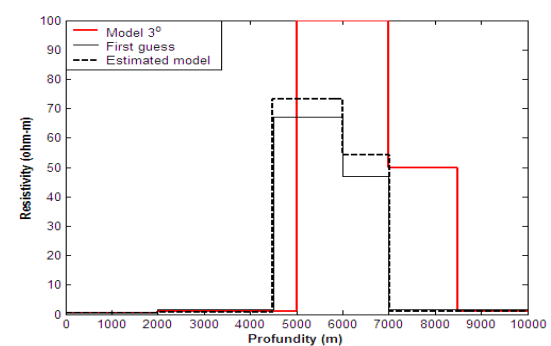

Figura 11: Resultado da inversão sobre o terceiro modelo geoelétrico (Fig.2), referente à minimização da função objetivo usual (Eq.3).

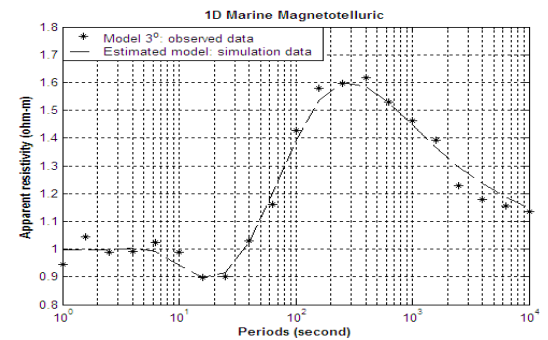

Figura 12: Resistividades aparentes do MMT $1 D$ simuladas tanto através do terceiro modelo geoelétrico (Fig.2), quanto pelo modelo estimado, referente à minimização da função objetivo normalizada (Eq.2).

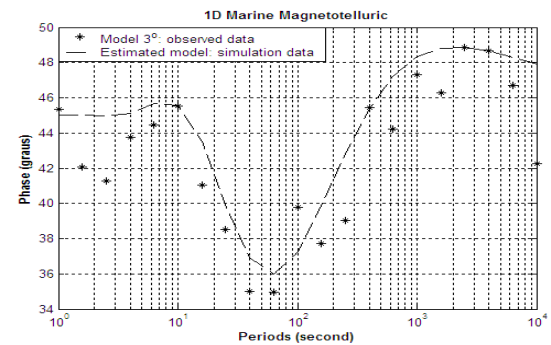

Figura 13: Fases do MMT $1 D$ simulados tanto através do terceiro modelo geoelétrico (Fig.2), quanto pelo modelo estimado, referente à minimização da função objetivo normalizada (Eq.2).

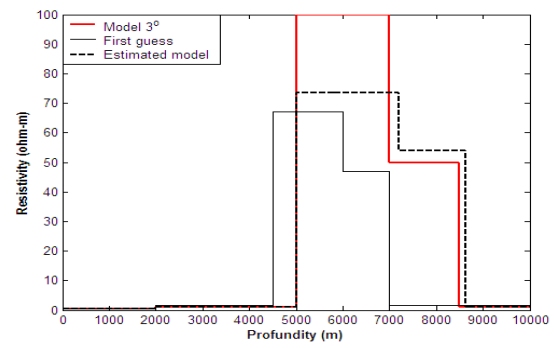

Figura 14: Resultado da inversão sobre o terceiro modelo geoelétrico (Fig.2), referente à minimização da função objetivo normalizada (Eq.2).

\section{Inversão do Quarto Modelo Geoelétrico}

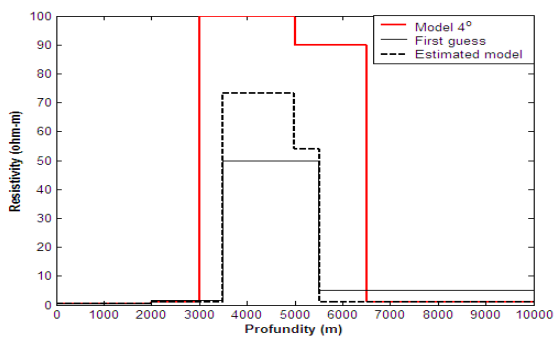

Figura 15: Resultado da inversão sobre o quarto modelo geoelétrico, referente à minimização da função objetivo usual (Eq.3).

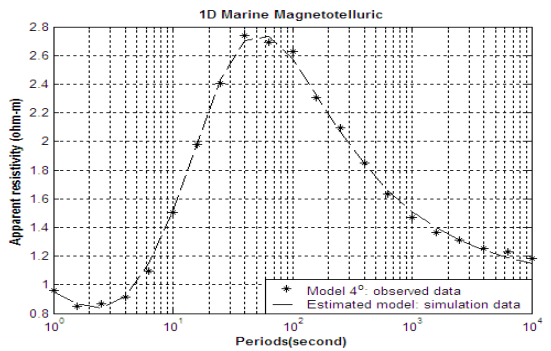

Figura 16: Resistividades aparentes do MMT $1 D$ simuladas tanto através do quarto modelo geoelétrico, quanto pelo modelo estimado, referente à minimização da função objetivo normalizada (Eq.2).

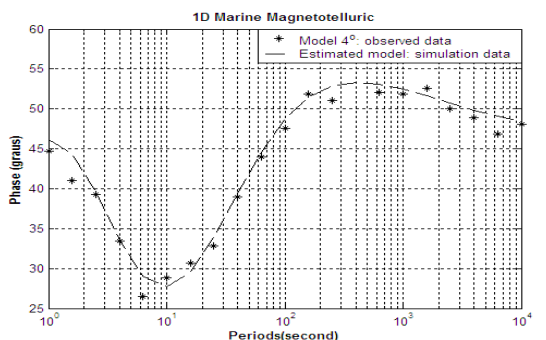

Figura 17: Fases do MMT 1D simulados tanto através do quarto modelo geoelétrico, quanto pelo modelo estimado, referente à minimização da função objetivo normalizada (Eq.2).

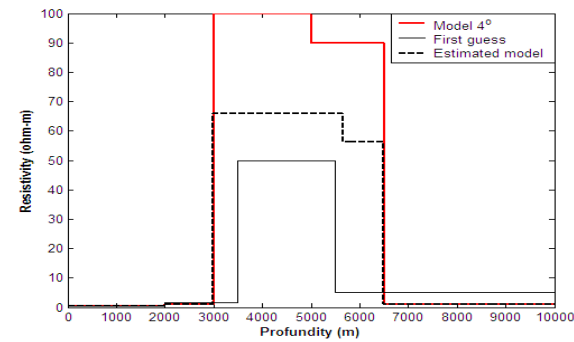

Figura 18: Resultado da inversão sobre o quarto modelo geoelétrico, referente à minimização da função objetivo normalizada (Eq.2). 
Referente à localização do topo da camada salina e a base da camada com hidrocarbonetos, observou-se um regular a bom delineamento quanto às referidas localizações segundo a minimização da função objetivo normalizada, Eq.(2), proposta por Almeida e Rijo (2011), Figs. $6,10,14$ e 18. Já segundo à minimização da função objetivo usual na literatura geofísica EM, Eq.(3) sobre os quatro processos de inversão em questão, observou-se um regular delineamento quanto à localização do topo da camada salina e a base da camada com hidrocarbonetos, Figs. 3, 7, 11 e 15 .

Agora, referente à localização do contacto entre a camada salina e a camada com hidrocarbonetos, observou-se nos quatro processos de reconstituição, Figs. 6, 10, 14 e 18, através da minimização da função objetivo normalizada, Eq.(2), um regular a bom delineamento sobre o referido contato. Já nos quatro processos de reconstituição, Figs. 3, 7, 11 e 15, através da minimização da função objetivo usual na literatura geofísica EM, Eq.(3), observou-se um regular delineamento do contato entre a camada de sal e a camada com hidrocarbonetos.

Quanto ao indicador de existência de camadas resistivas, ambos os processos de reconstituição acima mencionados, proporcionaram indicação de regular a boa, referente à existência de camadas mais resistivas, como é possível constatar nos modelos reconstituídos apresentados nas Figs. 3, 6, 7, 10, 11, 14, 15 e 18. Porém, foi observada a ausência de regularidade e/ou padrão quanto a melhor aproximação dos parâmetros de resistividades elétricas tanto das camadas salinas e quanto das camadas com hidrocarbonetos, sobre todos os modelos geoelétricos reconstituídos, tanto através da minimização da função objetivo usual na literatura geofísica, Eq.(3), quanto através da minimização da função objetivo normalizada, Eq.(2), Figs. 3, 6, 7, 10, 11, 14,15 e 18.

\section{Discussão e Conclusões}

Em todos os resultados de inversão apresentados não foram observadas boas a ótimas aproximações dos parâmetros de resistividades elétricas tanto das camadas salinas quanto das camadas com hidrocarbonetos e também foram constatadas regulares aproximações do contato camada salina e camada com hidrocarbonetos. Porém em todos os resultados foram verificadas a existência de camadas mais resistivas em relação aos sedimentos sobrejacentes e subjacentes. Em geral, em um processo de inversão geofísica EM, é muito difícil estabelecer o contato entre a camada salina e a camada com hidrocarbonetos, uma vez que ambas são altamente resistivas, resultando assim em um baixo contraste entre tais resistividades.

Os resultados, segundo a metodologia de inversão proposta por Almeida e Rijo (2011), (minimização da função objetivo, Eq.(2), juntamente com vínculos absolutos através do método de Marquartd), sobre modelos geoelétricos de ambientes altamente resistivos em águas profundas e ultra-profundas, melhor delineiam tanto o topo das camadas salinas, quanto a base das camadas com hidrocarbonetos, em relação aos resultados segundo à metodologia de inversão usual na literatura (minimização da função objetivo, Eq.(3), juntamente com vínculos absolutos através do método de Marquartd).

Uma hipótese a ser mais comprovada experimentalmente através da inversão geofísica de dados 1D do MMT é a seguinte: a ótima ou a regular recuperação dos parâmetros de resistividade elétrica das camadas relacionam-se com uma qualidade dos dados de fase do MMT 1D menos ruidosos ou mais ruidosos, respectivamente; como pôde ser constatado preliminarmente no primeiro parágrafo desta seção de discussão e conclusões. Já a ótima ou a regular recuperação do topo das camadas salinas e a base das camadas com hidrocarbonetos relacionam-se com uma qualidade dos dados de resistividades aparentes do MMT 1D menos ruidosos ou mais ruidosos, respectivamente; como pôde ser constatado preliminarmente no segundo parágrafo desta seção.

\section{Agradecimentos}

O autor agradece ao Conselho da Faculdade de Matemática e também ao Conselho do Campus Universitário de Castanhal da Universidade Federal do Pará pela aprovação do projeto de pesquisa, que a partir do qual foi possível o desenvolvimento deste trabalho.

\section{Referências}

Almeida, F.L de, Rijo, L., 2011. Modelagens Direta e Inversa de Dados mCSEM 3D. RBGf. v.29(2), p. 289-308.

Luiz, J. G., 1999. Informação a priori na inversão de dados Magnetotelúricos. Tese de Doutorado, UFPA. 92 p.

Medeiros, W. E. \& Silva, J. B. C., 1996. Geophysical inversion using approximate equality constrains. Geophysics, vol. 61, p. $1678-1688$.

Press, W. H., Teukolsky, S. A., Vetterling, W. T. and Flannery, B. P., 1992. Numerical Recipes in Fortran 77. Cambridge University Press. 2nd ed, p. 678-680.

Rijo, L., 2001. "Teoria dos Métodos Eletromagnético I, I/ e III ". UFPa, Departamento de Geofísica. ( www.rijo.pro.br, acesso: em novembro 2006 ).

Rijo, L., 2007. Cooperação Universidade-Indústria: O caso do método marine Controlled Source Electromagenetic (CSEM) na exploração de hidrocarbonetos. $10^{\text {th }}$ International Congress of the Brazilian Geophysical Society held in Rio de Janeiro, Brazil. 1 CD-ROM. 